\title{
Análisis de variables motivacionales y de estilos de vida saludables en practicantes de ejercicio físico en centros deportivos en función del género

\author{
Analysis of motivational variables and healthy lifestyles in sports center practitioners by gender \\ *Marta Leyton Román, *Judith García Matador, **Juan Pedro Fuentes García, **Ruth Jiménez Castuera \\ *Universidad Autónoma de Madrid (España), **Universidad de Extremadura (España)
}

\begin{abstract}
Resumen. Los objetivos de estudio fueron analizar las diferencias en función del género y del ámbito de pertenencia (rural-urbano) respecto a las variables analizadas en el presente trabajo, todo ello desde la Teoría de la Autodeterminación. La muestra estuvo formada por 202 sujetos de edades comprendidas entre 18 y los 64 años $(M=35.81$ y $D T=13.56)$, de los cuales 120 eran de género femenino y 82 de género masculino. Dicha muestra procedió tanto de zonas rurales (94 sujetos) como urbanas (108 sujetos) de Extremadura, constituida por practicantes en centros deportivos. Se aplicaron cinco cuestionarios, Regulación de la Conducta del Ejercicio Físico (BREQ-3), Escala de Satisfacción de las Necesidades Psicológicas Básicas en el ejercicio (PNSE), Motivos de Actividad Física, Intencionalidad para ser Físicamente Activo y Estilo de Vida Saludable. Respecto a la diferenciación de género, los hombres obtuvieron valores más elevados en cuanto a la necesidad psicológica básica de autonomía e intención de ser físicamente activo, mientras que las mujeres lo lograron en la necesidad psicológica básica de relaciones sociales y hábitos alimenticios. En el ámbito rural, la muestra obtuvo valores más elevados en cuanto a la forma de motivación más autodeterminada, la desmotivación, motivos de prácticas y variables de estilos de vida saludable referentes al consumo de tabaco y hábitos alimenticios que el ámbito urbano. Como conclusión, se determinó que un aumento de las formas de motivación más autodeterminadas a través de la satisfacción de las necesidades psicológicas básicas favorecerá los motivos de práctica referidos al disfrute y la competencia, consiguiendo así una adherencia a la práctica deportiva y estilos de vida más saludables.

Palabras claves: autodeterminación, motivación, necesidades psicológicas básicas, motivos de práctica, salud.
\end{abstract}

Abstract. The aim of the following report is to analyze gender and area (urban vs. rural) differences regarding the variables analyzed in this study using the Self Determination Theory as a framework. The sample was composed by two hundred and two (202) individuals aged between 18 and 64 ( $M=35.81$ and $D T=13.56), 120$ women and 82 men. This sample is composed by participants from rural (94 persons) and urban areas (108 persons) from Extremadura (South West of Spain). A criterion for inclusion was to be engaged in physical activities in gyms. We used five questionnaires: the Behavioral Regulation in Exercise Questionnaire (BREQ-3), the Psychological Need Satisfaction in Exercise Scale (PNSE), the Motives for Physical Activities Measure, the Motives for Physical Activities, and the Measurement of Intention to be Physically Active and Healthy Lifestyle Scale. Regarding gender comparison, men got higher scores in autonomy and intention of doing physical activities, which are variables of Psychological Need Satisfaction. On the other hand, women got high scores in social interaction and eating habits from the Psychological Need Satisfaction. Individuals from rural areas show higher scores than those from urban areas in self-directed motivation, demotivation, Motives for Physical Activities, and healthy lifestyle variables referred to smoking and eating habits. As a conclusion, an increase of self-directed motivation through Psychological Needs Satisfaction may enhance Motives for Physical Activities. This may lead to increased adherence to physical activity and healthier lifestyles. Keywords: self-determination, motivation, psychological need satisfaction, motives for physical activities, health.

\section{Introducción}

La motivación es el motor del comportamiento humano, indispensable para poder iniciar y desarrollar una actividad (Dosil, 2004), y es por ello que juega un papel de gran relevancia en la vida de los individuos, ya que permite comprender un gran número de conductas que aparecen tanto en el terreno educativo como en el deportivo, así como en el resto de ámbitos de la vida de las personas (Lomelí, López \& Valenzuela, 2016). Deci y Ryan (2000) afirman que la motivación es un elemento clave para conseguir la adherencia al deporte, ya que es el más importante e inmediato determinante del comportamiento humano.

Es un hecho, que la participación en actividades deportivas practicadas durante el tiempo de ocio aporta hábitos saludables y evitan el sedentarismo que caracteriza a nuestra sociedad actual (Beltrán-Carrillo, Devís-Devís \& Peiró-Velert, 2016).

La teoría de la autodeterminación (TAD) (Deci \& Ryan, 1985, 2000) es una macroteoría que defiende que los seres humanos son organismos activos, con tendencias innatas hacia el crecimiento y desarrollo psicológico, que se esfuerzan por dominar los desafíos e integrar sus experiencias en un sentido coherente, requiriendo refuerzos continuados y apoyos del ambiente social para funcionar eficazmente. De acuerdo con esta teoría, la motivación se organiza a lo largo de un continuo donde la menos autodeterminada es la desmotivación, que se refiere a la falta de intencionalidad del individuo por desempeñar alguna actividad. De este modo, se produce una falta de valoración de la activi-

Fecha recepción: 22-05-17. Fecha de aceptación: 19-12-17

Marta Leytón Román

marta.leyton@uam.es dad, así como un escaso sentimiento de identificación con ella. Le sigue la motivación extrínseca (determinada por agentes externos), que se desglosa en varias formas de regulación y, por último, la motivación intrínseca. Ésta es la más autodeterminada y la actuación es motivada por el placer y el disfrute que genera la propia actividad.

Según sus postulados, las necesidades psicológicas básicas o mediadores (autonomía, competencia y relaciones sociales), las cuales también vamos a analizar en este trabajo, son innatas, universales y esenciales para la salud y bienestar, algo natural de los seres humanos y, según los distintos grados de consecución de las mismas, se determinarán diferentes niveles de autodeterminación de la motivación del sujeto (Ryan \& Deci, 2000).

Para complementar la TAD, surgió el modelo jerárquico de la motivación (Vallerand, 1997; Vallerand \& Rousseau, 2001), que consideraba que existían tres niveles de motivación: a) el nivel global, que se refiere a la motivación presente habitualmente en la vida de las personas, puede ser entendido como un rasgo de las mismas; b) el nivel contextual, que corresponde a la motivación que se da en un contexto concreto, en nuestro caso, el contexto de los centros deportivos; yc) el nivel situacional, relacionado con la motivación que una persona posee en un momento y actividad determinados. Estos niveles se relacionan entre sí, pero suponen diferentes niveles de motivación. El modelo jerárquico de la motivación del Vallerand sustentado en la TAD, es clave para entender la adherencia o abandono del ejercicio físico (Puigarnau, Foguet, Balcells, Ambrós \& Anguera, 2016).

El modelo establece que los aspectos sociales del entorno influyen en la motivación en función de la consecución o no de una serie de necesidades psicológicas básicas (autonomía, competencia y relaciones sociales), cuya satisfacción incrementa el grado de motivación intrínseca (Deci \& Ryan, 2000; Ryan \& Deci, 2000). Al contrario, la falta de 
satisfacción de las mismas incrementa progresivamente la motivación extrínseca y, finalmente, la desmotivación, derivándose una serie de consecuencias a nivel afectivo, cognitivo y comportamental. Por tanto, esta teoría ha mostrado que, de forma general, la modificación de los determinantes (la manipulación de estructuras situacionales en los diferentes niveles de motivación) está relacionada con la modificación de la motivación y, como consecuencia, con la aparición de distintas consecuencias (Vallerand \& Rousseau, 2001).

En misma línea, Navarro et al. (2008), establecieron perfiles motivacionales en la práctica de ejercicio físico no competitivo, desde la TAD, determinando que el perfil más autodeterminado se asociaba a motivos de disfrute y fitness/salud siendo este más frecuente en chicas, y el menos autodeterminado se asoció con una menor frecuencia de práctica siendo más frecuente en chicos. Resultados similares encontraron González-Cutre, Sicilia y Águila (2011), determinando que las necesidades psicológicas básicas y las motivaciones más autodeterminadas se asociaban con motivos de salud y de ocio en la práctica de ejercicio físico, apareciendo el motivo de salud sólo en chicas, y no encontrando diferencia entre grupos de edad.

Si se quiere promocionar y educar para la salud, se debe tener presente los estilos de vida de las personas (Bonito, 2015). Existe un consenso universal que implica que todo lo relacionado con, nutrición adecuada (Galve, Alegría, Cordero, Fácila, Fernández \& Lluís-Ganella, 2014), prácticas de ejercicio físico traducido en acondicionamiento físico, e higiene personal realzan la salud o son protectoras de la misma (Casado-Pérez, Hernández-Barrera, Jiménez-García, Fernández-de-LasPeñas, Carrasco-Garrido \& Palacios-Ceña, 2015); y en contraposición el estrés, la falta de descanso, el tabaquismo, sedentarismo, ingesta excesiva de alcohol y/o de sustancias psicoactivas, suponen un riesgo para la misma (Moreno, Cruz \& Fonseca, 2014).

Unos buenos hábitos alimenticios, junto con un descanso regular y una práctica de actividad física regulary continuada, van a hacer que los practicantes adquieran un estilo de vida saludable (López, Montero, Mora \& Romero, 2014; Moreno, Diéguez, Lara \& Molina, 2015) y mejoren su desarrollo en todos los niveles (cognitivo, motor y afectivosocial). Será importante conocer la intención que tiene el individuo de ser físicamente activo ya que es una conducta beneficiosa que nos permitirá obtener estilos de vidas saludables acompañados de una buena alimentación y descanso. Investigaciones previas han indicado que la práctica de actividad física y deportiva influye sobre conductas de salud como la alimentación equilibrada entre otras (Jiménez, Cervelló, García, Santos \& Iglesias, 2007; Texeira et al., 2006; Johns, Hartmann-Boyce, Jebb \& Aveyard, 2014). Por lo tanto, es importante conocer la intención de ser físicamente activo, para ver la situación en la que se encuentran y promocionar la práctica de actividades para que pueda ser incluida en el hábito de vida.

Debido a la preocupación por mejorar la salud y prolongar la calidad de vida surgen gran cantidad de investigaciones donde muestran que la práctica de actividad física promovida por la motivación más autodeterminada promueve estilos de vida saludables a lo largo de toda la vida (Standage \& Gillison, 2007; Moreno, González-Cutre \& Cervelló, 2008; García-Calvo, Sánchez, Leo, Sánchez \& Amado, 2011; Leyton, Batista, Lobato, Aspano \& Jíménez, 2017; Sicilia, Ferriz-Morel \& Coll, 2016), por lo que si conocemos los motivos de prácticas podremos ofrecer a la población estilos de vida saludables (EVS) y formas de motivación más autodeterminadas.

Con respecto al sexo, Marcos, Borges, Rodríguez, Huescar y Moreno (2011), incidieron en que los motivos de práctica dependen de las características de los practicantes, añadiendo que la motivación integrada es clave para conseguir un mayor compromiso con la práctica. En sus resultaron encontraron que los chicos tienen un mayor motivo de afiliación y reconocimiento social que las chicas, además de reconocer que los motivos a menor edad están relacionados con la imagen, y a mayor edad prima el motivo de salud. Son varios los estudios(CastañedaVázquez, Zagalaz-Sánchez, Chacón-Borrego, Cachón-Zagalaz, \& Romero-Granados, 2014; Lamoneda \& Huertas, 2017; Práxedes, Sevil, Moreno, del Villar, \& García, 2016) que establecen que las chicas siguen mostrando menores niveles de participación en actividades físico deportivas que los chicos.

En cuanto al ámbito de la población (urbano-rural), McCartney et al. (2010), afirmó que los aspectos relacionados con el deporte y la actividad física durante el tiempo libre están condicionados por factores contextuales. Kafatos, Manios, Markatji, Giachetti, Vaz DeAlmeida, y Engstrom, (1999), ya determinaron que en las zonas urbanas la importancia que se da a la práctica deportiva durante el tiempo libre es mayor, observando una mayor motivación, interés y actitudes positivas hacia el mismo. Ellos lo denominaron una mayor «cultura deportiva». Por ello, se consideró adecuado analizar las variables motivacionales y estilos de vida saludables de practicantes de actividad físico deportiva tanto de zona rural como urbana de la comunidad de Extremadura, para ser capaces de ofrecer una amplia gama de posibilidades de prácticas accesible al mayor porcentaje de la población con los beneficios que esto conlleva.

Por lo tanto, el objetivo de este estudio fue analizar las diferencias en función del género y del ámbito de la población (urbano-rural) en las variables motivacionales (tipos de motivación y necesidades psicológicas básicas), los motivos de práctica y estilos de vida saludable en practicantes de ejercicio físico en centros deportivos.

En función del objetivo se plantearon las siguientes hipótesis:

- Basándonos en los estudios de Catañeda-Vázquez et al. (2014), Lamoneda y Huertas (2017) y Práxedes, et al. (2016), los varones mostrarán valores más elevados que las mujeres en las formas de motivación más autodeterminadas, en la satisfacción de las necesidades psicológicas básicas (autonomía, competencia y relaciones sociales) y en las variables de estilos de vida que favorecen la salud (hábitos alimenticios y la intención de ser físicamente activo).

- Basándonos en el estudio de Conesa, González, Valero-Valenzuela, García-Pallarés y De la Cruz-Sánchez (2016), las poblaciones urbanas mostrarán valores más elevados que las poblaciones rurales en las formas de motivación más autodeterminadas, satisfacción de las necesidades psicológicas básicas (autonomía, competencia y relaciones sociales) y en las variables de estilos de vida que favorecen la salud (hábitos alimenticios, hábitos de descanso y la intención de ser físicamente activo).

\section{Método}

\section{Diseño}

Se realizó un estudio descriptivo de corte transversal, en el cual no se interviene o manipulan las variables, sólo observándose lo que ocurre con ellas en condiciones naturales, en la realidad (Cubo-Delgado, Martín-Marín \& García-Ramos, 2011)

La técnica de muestreo utilizada fue probabilística-intencional ya que se escogieron los centros deportivos.

\section{Participantes}

La muestra del estudio estuvo formada por 202 sujetos, con una edad comprendida entre 18 y 64 años $(M=35.81$; $D T=13.53)$, procedentes de zonas rurales ( $\mathrm{N}=94$ sujetos; $M=39.29 ; D T=13.27$ ) y de zonas urbanas ( $\mathrm{N}=108$ sujetos; $M=32.70 ; D T=12.91$ ) de Extremadura. Participaron en el estudio 82 sujetos del sexo masculino $(M=31.14$ años; $D T=12.66)$, y 120 del género femenino ( $M=38.93$ años; $D T=$ 13.11). Todos ellos practicantes de centros deportivos, concretamente gimnasios que cuentan con actividades dirigidas.

\section{Instrumentos}

Para conocer las diferentes variables motivacionales, motivos de prácticas y EVS se administró una batería de cuestionarios siendo:

1) Escala de regulación de la conducta del ejercicio (BREQ-3), utilizado para medir desde la forma de motivación más autodeterminada a la menos autodeterminada. El cuestionario fue validado por Wilson, Rodgers, Loitz y Scime (2006). La versión traducida al español de este cuestionario se realizó por González-Cutre, Sicilia y Fernández (2010), permitiendo evaluar en contextos de actividad física todas las formas de 
motivación establecidas por la teoría de la autodeterminación. El instrumento, encabezado por «Yo hago ejercicio físico...», consta de 23 ítems donde se analizan seis variables. Las variables son cuatro para regulación intrínseca(Ej.: Porque creo que el ejercicio es divertido), cuatro para regulación integrada(Ej.: Porque está de acuerdo con mi forma de vida), tres para regulación identificada (Ej.: Porque valoro los beneficios que tiene el ejercicio físico), cuatro para regulación introyectada (Ej.: Porque me siento culpable cuando no lo práctico), cuatro para regulación externa (Ej.: Porque los demás me dicen que debo de hacerlo) y cuatro para desmotivación (Ej.: No veo por qué tengo que molestarme en hacer ejercicio)

2) Escala de medición de la satisfacción de las necesidades psicológicas básicas en el ejercicio (PNSE), evalúa las necesidades psicológicas básicas cuyo cuestionario fue validado por Wilson, Rogers, Rodgers y Wild (2006). La traducción de este cuestionario al español se realizó por Navarro et al. (2008). El cuestionario se compone de 18 ítems encabezado por «En mis entrenamientos...» detectando tres variables de las necesidades psicológicas básicas: autonomía (Ej.: «Siento que puedo hacer ejercicios a mi manera»), competencia (Ej.: «Tengo confianza para hacer los ejercicios más desafiantes.») y relaciones sociales (Ej.: «Me siento relacionado con los que me relaciono porque hacemos ejercicios juntos»).

3) Escala de Medida de los Motivos para la Actividad física (MPAM), validado por Moreno, Cervelló y Martínez (2007), fue diseñado para medir los motivos de práctica. Cuestionario de 30 ítems que mide los motivos de práctica hacia la actividad física agrupados en cinco variables: disfrute (Ej.: Porque es divertido), apariencia (Ej.: Porque quiero mantener mi peso para tener buena imagen), social (Ej.: Porque me gusta estar con mis amigos, fitness/salud (Ej.: Porque quiero estar en buena forma física) y Competencia (Ej.: Porque quiero desarrollar nuevas habilidades).

4) Cuestionario de estilos de vida (EVS), mide los hábitos saludables de los practicantes pertenecientes a las variables de estilos de vida, nos hemos apoyado en la versión traducida al castellano por Moreno, Jiménez y Gil (2011) del cuestionario diseñado por Wold(1995) basada en 20 ítems que hacen referencia a cuatro variables: alimentación equilibrada, que consta de cinco ítems (Ej.: «Normalmente tomo pescado dos o más veces por semana»), respeto al horario de las comidas, con cinco ítems (Ej.: «Normalmente desayuno, como y ceno a la misma hora»), hábitos de descanso, constituido por cinco ítems (Ej.: «Duermo el número de horas suficiente para que mi cuerpo esté descansado») y consumo de tabaco con cinco ítems (Ej.: «Fumo de forma habitual»).

5) Cuestionario de medida de la intencionalidad de ser físicamente activo (MIFA) para poder medir la intención de practicar actividad físico-deportiva que poseía cada sujeto de la muestra, se utilizó la Escala de Medida de la Intencionalidad para ser Físicamente Activo (Hein, Müür \& Koka, 2004), utilizada por Moreno, Moreno, y Cervelló (2007), la cual consta de cinco ítems que se agrupan en un mismo factor o variable, por lo que miden el mismo aspecto (Ej.: «Me interesa el desarrollo de mi forma física»). Los ítems van precedidos de la frase «Respecto a tu intención de practicar alguna actividad físico-deportiva...».

Las respuestas de todos los cuestionarios están formuladas en una escala tipo Likert en la que cada ítem tiene un rango de respuesta de 1 a 5. El 1 corresponde a totalmente en desacuerdo y el 5 a totalmente de acuerdo con la formulación de la frase.

\section{Procedimiento}

Definido el objeto de estudio, primeramente, seleccionamos los instrumentos y elaboramos un dossier con datos de interés como la población, edad, y sexo. Posteriormente, se contactó con los centros deportivos para la cumplimentación de los cuestionarios exponiendo objetivo principal del estudio, con el fin de que mostraran mayor interés al completar los mismos. Por otra parte, una vez cumplimentado el consentimiento informado para participar en el estudio, se les animó a que contestaran los cuestionarios de la manera más sincera posible, indicándoles que eran anónimos y confidenciales.
El tiempo empleado para cumplimentar cada cuestionario fue de unos 10 minutos, aproximadamente, dependiendo de la edad, capacidad comprensiva y velocidad de lectura del sujeto.

\section{Análisis de Datos}

El programa utilizado para analizar los datos obtenidos en los diferentes cuestionarios es el programa estadístico IBM SPSS Statistics 19.0. Se realizó el análisis factorial y el análisis de fiabilidad, para comprobar la consistencia interna de los cuestionarios. Seguidamente, se creó las variables de aquellos factores en los que la fiabilidad sea aceptable. Tras la realización de la prueba de normalidad de KolmogorovSmirnov y homogeneidad de varianzas mediante el test de Levene, cabe indicar que los resultados obtenidos de ambos tests muestran una distribución normal de los datos.

A continuación, se realizaron análisis descriptivos, análisis de varianza realizando el ANOVA en función del sexo y población.

\section{Resultados}

\section{Estadísticos descriptivos y análisis de fiabilidad}

Nuestro estudio mostró la consistencia interna de los datos en las variables e instrumentos utilizados, mediante el análisis de fiabilidad, observándose que tan solo fue descartado un factor del cuestionario de EVS (hábitos de descanso) por presentar un Alpha de Cronbach inferior al recomendado (.70) (Nunnally, 1978). En el resto de variables, dado el pequeño número de ítems que componen los factores (tres o cuatro para cada factor), la consistencia interna algo menos a .70 observada puede ser marginalmente aceptada (Hair, Anderson, Tatham \& Black, 1998; Nunnally \& Bernstein, 1994).

Tal y como observamos en las medias, los valores motivacionales más elevados son la regulación intrínseca (4.26) e identificada (4.45). Respecto a los factores de las necesidades psicológicas aparecen la competencia (3.98) y la autonomía (3.73). En cuanto a los motivos de práctica, encontramos fitness/salud (4.53) y disfrute (4.28) y, por último, la intención de ser físicamente actico (4.53) y los hábitos alimenticios (4.13) en los estilos de vida saludable (tabla 1).

\begin{tabular}{|c|c|c|c|}
\hline Variable & Media & Desv. Típica & Alpha de Cronbach \\
\hline \multicolumn{4}{|c|}{$\begin{array}{l}\text { Regulación de la conducta } \\
\text { del ejercicio }\end{array}$} \\
\hline Reg. Intrínseca & 4.26 & 0.87 & .89 \\
\hline Reg. Integrada & 4.04 & 1.02 & .87 \\
\hline Reg. Identificada & 4.45 & 0.64 & .70 \\
\hline Reg. Introyectada & 2.01 & 0.95 & .67 \\
\hline Reg. externa & 1.41 & 0.80 & .81 \\
\hline Desmotivación & 4.25 & 0.67 & .78 \\
\hline \multicolumn{4}{|c|}{ Necesidades psicológicas en el ejercicio } \\
\hline Autonomía & 3.73 & 0.99 & .89 \\
\hline Competencia & 3.98 & 0.92 & .90 \\
\hline Relaciones & 3.48 & 1.08 & .85 \\
\hline \multicolumn{4}{|l|}{ Motivos de práctica } \\
\hline M. Disfrute & 4.28 & 0.75 & .87 \\
\hline M. Apariencia & 3.95 & 0.83 & .82 \\
\hline M. Social & 3.55 & 1.09 & .84 \\
\hline M. Fitness/Salud & 4.53 & 0.49 & .80 \\
\hline M. Competencia & 3.80 & 1.05 & .89 \\
\hline \multicolumn{4}{|c|}{ Estilos de vida saludables } \\
\hline Tabaco & 1.51 & 1.02 & .90 \\
\hline Háb. Alimenticios & 4.13 & 0.80 & .83 \\
\hline Intención & 4.53 & 0.55 & .70 \\
\hline
\end{tabular}

\section{Análisis de varianza en función del sexo y población.}

Las variables que fueron significativas en función del sexo, correspondientes a una significatividad menor que .05 , son tal y como observamos en la tabla 2 las variables motivación identificada (.00), motivación introyectada (.00), motivación extrínseca (.00), autonomía (.02), relaciones sociales (.00), apariencia (.00), motivos de práctica sociales (.00), fitness/salud (.00), hábitos alimenticios (.00) e intención de ser físicamente activo (.03).

Dentro de estas variables que han sido significativas, podemos ver que tanto en la motivación identificada, relaciones sociales, apariencia, motivos sociales y fitness/salud así como así como los hábitos alimenticios, la media es mayor en las mujeres que en los hombres. Por el 
Tabla 2

Análisis de varianza entre las diferentes variables como variables dependientes y el género como independiente.

\begin{tabular}{lcc}
\hline Vénero como independiente. & Media Cuadrática & $F$ \\
\hline Reg. Intrínseca & 1.59 & 2. \\
Reg. Integrada & .25 & .24 \\
Reg. Identificada & 4.00 & 10.05 \\
Reg. Introyectada & 6.98 & 7.90 \\
Reg. Extrínseca & 5.77 & 9.35 \\
Desmotivación & .442 & .97 \\
Autonomía & 5.39 & 5.5 \\
Competencia & 1.02 & 1.2 \\
Relaciones & 10.37 & 9.0 \\
M. Disfrute & 1.61 & 2. \\
M. Apariencia & 5.14 & 7.5 \\
M. Social & 17.98 & 16 \\
M. Fitness/Salud & 4.16 & 18.28 \\
M. Competencia & .05 & .04 \\
Tabaco & .04 & .04 \\
Háb. alimenticios & 10.63 & 17.82 \\
Intención & 1.3 & 4.67
\end{tabular}

Intención

\begin{tabular}{llllll} 
& 1.3 & 4.67 & .03 & 4.46 & 4.63 \\
\hline
\end{tabular}

isicamente activo.

: significativo entre .00 y .05

Tabla 3.

Análisis de varianza entre las diferentes variables como variables dependientes y la población considerándola como independiente.

\begin{tabular}{lccccc}
\multicolumn{6}{l}{ población considerándola como independiente. } \\
\hline Variable & Media Cuadrática & $F$ & $p$ & Media rural & Media urbano \\
\hline Reg. Intrínseca & 7.17 & 9.83 & .00 & 4.46 & 4.09
\end{tabular}

Reg. Integrada

Reg. Identificada

Reg. Introyectad

Reg. Extrínseca

Desmotivación

Autonomía

Competencia

Relaciones

M. Disfrute

M. Disfrute
M. Apariencia

M. Apariencia
M. Social

M. Social

M. Fitness/Salud

M. Comp
Tabaco

Háb. alimenticio

Háb. alimentc

Intención

Nota. Reg.: regulación; M: motivo de práctica; Háb: hábitos; Intención: Intención de ser

$p$ : significativo entre .00 y .05

contrario, las medias más elevadas en los hombres que en las chicas encontramos motivación introyectada y externa, la autonomía y la intención de ser físicamente activo.

Para el segundo caso, se llevó a cabo un análisis de varianza en función de la edad, donde la variable independiente fue la edad y las dependientes los factores de la motivación autodeterminada (motivación intrínseca, integrada, identificada, introyectada, extrínseca y desmotivación), los factores de necesidades psicológicas básicas (autonomía, competencia y relaciones sociales), los factores de motivos de práctica (disfrute, apariencia, social, fitness/salud y competencia) y los factores de los estilos de vida (consumo de tabaco, hábitos alimenticios e intención de ser físicamente activo) (tabla 2).

En la tabla 3 obtenemos las diferencias significativas en función de la población siendo las variables de motivación intrínseca, identificada, relaciones sociales, motivos de práctica de disfrute, apariencia, social, fitness/salud y hábitos alimenticios (.00), introyectada (.01), desmotivación (.03) y consumo de tabaco (.04).

Dentro de estas variables que han sido significativas, podemos observar que las medias más elevadas afectan a la población rural en factores como motivación intrínseca, identificada, introyectada, desmotivación, relaciones sociales, factores de motivos de práctica (disfrute, apariencia, social y fitness/salud), consumo de tabaco y hábitos alimenticios. La única media más elevada es en la población urbana con el factor de regulación introyectada (tabla 3).

\section{Discusión}

Dada la importancia del análisis de la motivación en la práctica deportiva ya sea en contextos educativos, competitivos y no competitivos, el propósito de este estudio fue analizar las diferencias en función del sexo y la zona de pertenencia de diferentes centros deportivos de Extremadura.

En relación a la primera hipótesis que indicaba que los varones mostrarán valores más elevados que las mujeres en las formas de motivación más autodeterminadas, en la satisfacción de las necesidades psi- cológicas básicas (autonomía, competencia y relaciones sociales) y en las variables de estilos de vida que favorecen la salud (hábitos alimenticios y la intención de ser físicamente activo), se puede determinar que la hipótesis no se cumple. Esto se debe a que, en los resultados encontrados en las diferencias entre hombres y mujeres, éstos presentaron medias más altas en las variables regulación introyectada, regulación extrínseca, necesidades psicológicas básicas de autonomía y competencia, motivo de competencia, consumo de tabaco e intención de ser físicamente activo. Sin embargo, no se encontraron diferencias significativas ni medias más altas con respecto a las mujeres, en las formas de motivación más autodeterminadas, en la necesidad psicológica de relaciones sociales, así como en las variables hábitos alimenticios e intención de ser físicamente activo, tal y como se planteaba en la primera hipótesis.

Lamoneda y Huertas (2017), en su estudio con adolescentes, determinaron que son más las chicos que practican actividad físico-deportiva, sin embargo también concluyeron que éstos prefieren el exterior para su práctica. Este hecho podría sugerir que los participantes masculinos de nuestra muestra no presenten las formas de motivación descritas en la hipótesis, al pertenecer a centros deportivos.

Autores como Moreno, Cervelló y González-Cutre (2007) señalaron que el sexo masculino puntuaba más alto en competencia percibida cuando realizaba deporte que el género femenino y éste, en cambio, puntuaba más alto en la satisfacción de relacionarse con los demás. Así, Ntoumanis (2001) también aporta en su estudio que las mujeres tuvieron valores mayores que los hombres en la relación con los demás. Según López, Irisarri y Fernández (2017), en las mujeres entre 50 y 70 años, el apoyo social resulta necesario para alcanzar unos niveles saludables de AF. Esto apoya los resultados encontrados en este estudio, donde se observó que la media en las relaciones sociales fue mayor en mujeres que en los hombres.

Estudios como el llevado a cabo por Ntoumanis (2001) o el realizado por Rodríguez, Mata y Ruiz (2006), mostraron que el sexo masculino mostraba mayores puntuaciones en competencia percibida que el femenino, al igual que ocurre en nuestro estudio, aunque la diferencia encontrada con respecto a las mujeres no fue muy grande.

En la misma línea encontramos más estudios que muestran cómo las mujeres dan más importancia a ciertos motivos de práctica tales como la apariencia, mientras que los hombres puntuarían más alto en motivos asociados a la competencia (Furnham, Badmin \& Sneade, 2002; Kilpatrick, Hebert \& Bartholomew, 2005; Sicilia, González-Cutre, Artés, Orta, Casimiro \& Ferriz, 2014; Cambronero, Blasco-Mira, Chiner \& Lucas-Cuevas, 2015). Resultados similares fueron encontrados en este estudio, donde las mujeres puntúan más alto en el motivo de apariencia y los hombres en los motivos asociados a la competencia. Además, en un estudio reciente, Moreno, Pardo, Jorge, y Huéscar (2016), determinaron que el motivo salud tenía valores medios más altos en mujeres entre los 18 y los 36 años, seguido del motivo imagen y desarrollo de la habilidad. Al igual ocurre en nuestro estudio con los motivos de salud e imagen.

González-Cutre y Sicilia (2012), sugirieron que personas que participan en actividades supervisadas por un monitor tendían a presentar menos síntomas de adeherencia hacia el ejercicio que los que practican por su cuenta. Sin embargo, investigaciones más recientes como la de Moreno, Sicilia, Sáenz-López, González-Cutre, Almagro y Conde (2015), determinan que la interacción entre el monitor y el practicante puede servir para la transmisión de valores y hábitos saludables, apoyando la creación de un clima motivacional adecuado por parte del monitor, para que la práctica de actividad físico-deportiva sea una actividad agradable y motivante. Esto está en concordancia con nuestro estudio, donde las medias más altas de motivación se observaron en la regulación intrínseca, siendo mayores en mujeres y habiendo sólo un.18 de diferencia entre hombres y mujeres, lo que les llevaría a tener una mayor intención de ser físicamente activas. Como se ha comentado la motivación intrínseca supone realizar una actividad por el propio disfrute y placer que supone su realización, generando consecuencias positivas (Vallerand \& Rousseau, 2001) como los motivos de práctica de disfrute, apariencia, relaciones sociales y fitness/salud, tal y como apa- 
rece en nuestro estudio.

En relación a la segunda hipótesis que determinaba que las poblaciones urbanas mostrarán valores más elevados que las poblaciones rurales en las formas de motivación más autodeterminadas, satisfacción de las necesidades psicológicas básicas (autonomía, competencia y relaciones sociales) y en las variables de estilos de vida que favorecen la salud (hábitos alimenticios, hábitos de descanso y la intención de ser físicamente activo), podemos determinar que la hipótesis no se cumple. Esto de debe a que, los resultados encontrados referente a las diferencias entre la población rural y urbana, mostraton unas medias más elevadas en la población urbana en factores como regulación introyectada y necesidad psicológica básica de autonomía. Sin embargo, no se encontraron diferencias significativas ni medias más altas, con respecto a la población rural, en el resto de variables que establece la segunda hipótesis.

A pesar de no ser muchos los estudios existentes que diferencien la población rural y urbana española, Sicilia, Águila, Muyor, Orta, y Moreno (2009), tras su estudio de perfiles motivacionales en centros deportivos municipales, resalta la importancia de crear entornos que favorezcan una competencia mayor y tipos de motivación autodeterminada, dada la asociación que estas variables mantuvieron con niveles altos de experiencia autotélica percibida, y favorecer la adherencia a la práctica de actividad física. En nuestro estudio observamos resultados similares donde la población rural mantiene la práctica de actividad física con valores elevados de motivación más autodeterminada, en comparación con la población urbana que presentó valores más bajos. Este hecho va en concordancia con el hecho de que en la población rural se obtuvieron mayores medias en todos los motivos de práctica analizados, generando, por lo tanto, esta mayor motivación intrínseca hacia la práctica consecuencias positivas.

En un estudio más reciente, Conesa, et al. (2016), determinaron que el porcentaje de practica de actividad física era menor en zonas rurales que en zonas urbanas, en una población de 29.478 adultos españoles, concluyendo que estos datos podían ser debido a falta de instalaciones y recursos principalmente. Motivo por el cual se planteó la segunda hipótesis. Sin embargo, según los datos obtenidos en nuestro estudio la motivación más autoderminada, las necesidades psicológicas básicas de competencia y relaciones sociales, así como todos los motivos de práctica analizados fueron mayores en la población rural. Hecho que nos obliga a analizar más profundamente ambas poblaciones en futuros estudios, como es el uso de instalaciones, las ofertas de práctica, y muy importante aumentar la muestra de ambas poblaciones.

\section{Conclusiones}

Consideramos importante este estudio ya que nos sirve para aumentar la importancia de establecer estrategias motivacionales para la mejora de la motivación intrínseca en hombres que realizan ejercicio físico en centros deportivos, así como de la población urbana. Por ejemplo, a través de la satisfacción de la necesidad psicológica básica de relaciones sociales, planteando actividades con diferentes tipos de agrupamiento y trabajo cooperativo.

Algunos de los futuros estudios que planteamos serían aplicar programas multidisciplinar, en la que no sólo se realice actividad física, sino un programa de desarrollo integral, donde también exista un aporte cognitivo, afectivo y social para que el usuario aprenda conceptos relacionados con la práctica, con los beneficios que ésta produce experimentando estilos de vida saludables. Y cómo aspecto más importante, realizar un estudio de intervención que analice las variables que fomentan estilos de vida saludables.

Se concluye que se encontraron en los hombres valores más elevados que en las mujeres en las variables regulación introyectada, regulación extrínseca, necesidades psicológicas básicas de autonomía y competencia, motivo de competencia, consumo de tabaco e intención de ser físicamente activo; $\mathrm{y}$ en los participantes pertenecientes al ámbito urbano obtienen valores más elevados que en el ámbito rural, en los factores regulación introyectada y necesidad psicológica básica de autonomía.
Se hace necesaria la elaboración de estrategias específicas para los hombres practicantes de ejercicio físico en centros deportivos, así como un análisis más profundo de los motivos de la práctica de ejercicio físico en poblaciones urbanas, con objetivo de mejorar la motivación intrínseca en estas poblaciones, a través de la satisfacción de las necesidades psicológicas básicas, en especial de la necesidad de relaciones sociales. En posteriores estudios sería necesario ampliar la muestra y realizar comparaciones con sujetos que realicen ejercicio físico fuera de centros deportivos.

En relación a los resultados hallados, sería necesaria una encuesta inicial sobre las preferencias de los usuarios de centros deportivos, sobre todo en zonas urbanas donde, debido al número de población, las personas se conocen menos, para poder dirigirles hacia las prácticas que más les interese, y así guiarles hacia actividades grupales que les guste y fomentes la adherencia a la práctica de ejercicio físico.

\section{Referencias}

Beltrán-Carrillo, V. J., Devís-Devís, J., \& Peiró-Velert, C. (2016). Actividad física y sedentarismo en adolescentes de la Comunidad Valenciana. Revista Internacional de Medicina y Ciencias de la Actividad Física y del Deporte, 12(45), $122-137$

Bonito, J. (2015). La educación para la salud en la actualidad: algunas breves notas. Atención Primaria, 47(1), 32-37.

Cambronero, M., Blasco-Mira, J. E., Chiner, E., \& Lucas-Cuevas, Á. G. (2015). Motivos de participación de los estudiantes universitarios en actividades físicodeportivas. Revista Iberoamericana de Psicología del Ejercicio yel Deporte, 10(2), 179-186.

Casado-Pérez, C., Hernández-Barrera, V., Jiménez-García, R., Fernández-de-LasPeñas, C., Carrasco-Garrido, P., \& Palacios-Ceña, D. (2015). [Physical activity in adult working population: Results from the European National Health Survey for Spain(2009).]. Atención primaria/Sociedad Española de Medicina de Familia y Comunitaria, 47(9), 563-572. doi: 10.1016/j.aprim.2015.01.005

Castañeda-Vázquez, C., Zagalaz-Sánchez, M. L., Chacón-Borrego, F., CachónZagalaz, J., \& Romero-Granados, S. (2014). Características de la práctica deportiva en función del género. Estudiantes de la Facultad de Ciencias de la Educación: Universidad de Sevilla. Retos. Nuevas tendencias en educación física, deporte y recreación, 25, 63-67.

Conesa, E., González, A., Valero-Valenzuela, V., García-Pallarés, J., \& De la CruzSánchez,E. (2016). Indicadores climáticos y geográficos asociados a la práctica de actividad física duranteel tiempo libreen lapoblación adulta española. Journal of Sport and Health Research, 8(2), 93-102.

Cubo-Delgado, S., Martín-Marín, B., \& García-Ramos, J. L. (2011). Métodos de investigación y análisis de datos en ciencias sociales y de la salud. Madrid: Ediciones Pirámide Grupo Anaya, S. A.

Deci, E. L. \& Ryan, R. M. (1985). Intrinsic motivation and self-determination in human behavior. New York: Plenum Press.

Deci, E. L. y Ryan, R. M. (2000). The «what» and «why» of goal pursuits: Human needs and the self-determination of behavior. Psychological Inquiry, 11(4), 227268. https://doi.org/10.1207/S15327965PLI1104_01

Dosil, J. (2004). Psicología de la Actividad Física y del Deporte. Madrid. McGraw Hill.

Furnham,A., Badmin, N., \& Sneade, I. (2002). Body image dissatisfaction: Gender differences in eating attitudes, self-esteem, and reasons for exercise. The Journal of Psychology, 136(6), 581-596. https://doi.org/10.1080/00223980209604820

Galve, E., Alegría, E., Cordero, A., Fácila, L., Fernández, J., \& Lluís-Ganella, C. (2014). Temas de actualidad en cardiología: riesgo vascular y rehabilitación cardiaca. Revista Española de Cardiología, 67(3), 203-210. https://doi.org/ 10.1016/j.recesp.2013.09.021

García-Calvo, T., Sánchez, P. A, Leo, F. M., Sánchez, D., \& Amado, D. (2011). Incidencia de la Teoría de la Autodeterminación sobre la práctica deportiva. Revista internacional de ciencias del deporte, 7(25), 266-276. doi:10.5232/ ricyde2011.02502

González-Cutre, D. \& Sicilia, Á. (2012). Motivation and exercise dependence:A study based on self-determination theory. Research Quarterly for Exercise and Sport, 83(2), 318-329. https://doi.org/10.1080/02701367.2012.10599863

González-Cutre, D., Sicilia, A., \& Águila, C. (2011). Interplay of different contextual motivations and their implications for exercise motivation. Journal of Sport Science and Medicine, 10(2), 274-282.

González-Cutre, D., Sicilia, Á., \& Fernández,A. (2010). Hacia una mayor comprensión de la motivación en el ejercicio físico: medición de la regulación integrada en el contexto español. Psicothema, 22(4), 841-847.

Hair, J. F., Anderson, R. E., Tatham, R. L., \& Black, W. C. (1998). Multivariate Data Analysis. Upper Saddle River, NJ: Prentice-Hall. 
Hein, V., Müür, M., \& Koka,A. (2004). Intention to be physically active after school graduation and its relationship to three types of intrinsic motivation. European Physical Education Review, 10(1), 5-19. https://doi.org/10.1177/ 1356336X04040618

Jiménez, R., Cervelló, E., García, T., Santos, F. J., \& Iglesias, D. (2007). Estudio de las relaciones entre motivación, práctica deportiva extraescolar y hábitos alimenticios y de descanso en estudiantes de Educación Física. International Journal of Clinical and Health Psychology, 7(2), 385-401.

Johns, D. J., Hartmann-Boyce, J., Jebb, S. A., \& Aveyard, P. (2014). Diet or Exercise Interventions vs Combined Behavioral Weight Management Programs: A Systematic Review and Meta-Analysis of Direct Comparisons. Journal of the Academy of Nutrition and Dietetics, 114(10), 1557-1568. https://doi.org/ 10.1016/j.jand.2014.07.005

Kafatos, A., Manios, Y., Markatji, I., Giachetti, I., de Almeida, M. D. V., \& Engstrom, L. M. (1999). Regional, demographic and national influences on attitudes and beliefs with regard to physical activity, body weight and health in a nationally representative sample in the European Union. Public health nutrition, 2(1a), 87-96. https://doi.org/10.1017/S1368980099000130

Kilpatrick, M., Hebert, E., \& Bartholomew, J. (2005). College students'motivation for physical activity: Differentiating men's and women's motives for sport participation and exercise. Journal of American College Health, 54(2), 84-94. https://doi.org/10.3200/JACH.54.2.87-94

Lamoneda, J. \& Huertas, F. J. (2017). Análisis de la práctica deportiva-recreativa a través de un programa de promoción en el recreo en función del sexo en adolescentes españoles. RETOS. Nuevas Tendencias en Educación Física, Deportey Recreación, 32, 25-29.

Leyton, M., Batista, M., Lobato, S., Aspano, M‥ I., \& Jíménez, R. (2017). Application of two intervention programs in order to optimize motivation and eating habits in adults and the elderly. Jounal of Human Kinetics, 59(1), 131 142. https://doi.org/10.1515/hukin-2017-0153

Lomelí, A. M., López, M. G., \& Valenzuela, J. R. (2016). Autoestima, motivación e inteligencia emocional: Tres factores influyentes en el diseño exitoso de un proyecto de vida de jóvenes estudiantes deeducación media. Revista Electrónica Educare, 20(2), 29-29. http://dx.doi.org/10.15359/ree.20-2.4

López, F. L., Irisarri, M. A. G., \& Fernández, M. J. S. (2017). The correlates of physical activity among the population aged $50-70$ years (Determinantes de la actividad física entre las personas de 50 a 70 años). RETOS, Nuevas Tendencias en Educación Física, Deportey Recreación, 31(1), 181-187.

López, M. R., Montero, M. P., Mora, A. I., \& Romero, J. F. (2014). Hábitos de alimentación, salud y género en personas mayores. European Journal of investigation in health, psychology and education, 4(1), 31-40.

Marcos, P., Borges, F., Rodríguez, A., Huescar, E., \& Moreno, J. A. (2011) Indicios de cambio en los motivos de práctica físico deportiva según el sexo y la edad. Apuntes de Psicología, 29(1), 123-132.

McCartney, G., Thomas, S., Thomson, H., Scott, J., Hamilton, V., Hanlon, P., Morrison, D., \& Bond, L. (2010). The health and socioeconomic impacts of major multi-sport events: systematic review (1978-2008). BMj, 340, 1-9. doi:10.1136/bmj.c2369.

Moreno, J., Cruz, H., \& Fonseca, A. (2014). Evaluación de razones de prevalencia para sedentarismo y factores de riesgo en un grupo de estudiantes universitarios. Chía, Colombia. Enfermería Global: Revista Electrónica Semestral de Enfermería, 13(2), 114-122.

Moreno, V., Diéguez, I., Lara, J. J., \& Molina, G. (2015). Estado nutricional, hábitos nutricionales, calidad de vida y somnolencia diurna en personal de ocio nocturno de Córdoba. Nutrición Hospitalaria, 31(4), 1778-1786. doi:10.3305/ nh.2015.31.4.8506

Moreno, B., Jiménez, R., \& Gil,A. (2011). Análisis de las necesidades psicológicas básicas y los hábitos saludables en mujeres cacereñas adultas y mayores. Comunicación presentada en el IV Congreso Internacional deActividad Físico Deportiva para Mayores, Málaga, España.

Moreno, J. A., Cervelló, E., \& Martínez, A. (2007). Validación de la Escala de Medida de los Motivos para la Actividad Física Revisada en español: Diferencias por motivos de participación. Anales de Psicología, 23(1), 167-176.

Moreno, J. A., Moreno, R., \& Cervelló, E. (2007). El autoconcepto físico como predictor de la intención de ser físicamente activo. Psicología y Salud, 17(2), 261-267.

Moreno, J. A., Cervelló, E., Vera, J. A., \& Ruiz, L. M. (2007). Physical SelfConcept of Spanish schoolchildren: differences by gender, sport practice and levels of sport involvement. Journal of Education and Human Development, 1(2), 1-17.

Moreno, J.A., González-Cutre, D., \& Cervelló, E. (2008).Motivación y salud en la práctica físico-deportiva: diferencias según el consumo de alcohol y tabaco. International Journal of Clinical and Health Psychology, 8(2), 483-494.

Moreno, J. A., Pardo, M., Jorge, P., \& Huéscar, E. (2016). Motivos de práctica físico-deportiva en mujeres. Revista de Psicología del Deporte, 25(1), 35-41.

Moreno, J. A., Sicilia,A., Sáenz-López, P., González-Cutre, D., Almagro, B. J., \&
Conde, C. (2015). Análisis motivacional comparativo en tres contextos de actividad física. Revista Internacional de Medicina y Ciencias de la Actividad Física y del Deporte, 14(56), 665-685.

Navarro, N., González-Cutre, D., Marcos, P. J., Borges, F., Hernández, A., Vera, J. A., \& Moreno, J. A. (2008). Perfiles motivacionales en la actividad física saludable: un estudio desde la perspectiva de la teoría de la autodeterminación. EnActas del XI Congreso Nacional, XI Andaluz y III Iberoamericano de Psicología de la Actividad Física y del Deporte. Sevilla: Universidad Pablo de Olavide.

Ntoumanis, N.(2001).Aself-determinationapproach to theunderstanding of motivation in physical education. British Journal of Educational Psychology, 71(2), 225242. doi: 10.1348/000709901158497

Nunnally, J. C. (1978). Psychometric theory. New York: McGraw-Hill.

Nunnally, J. C. \& Bernstein, I. H. (1994). Psychometric Theory. Nueva York: McGraw-Hill.

Práxedes, A., Sevil, J., Moreno, A., del Villar, F., \& García, L. (2016). Niveles de actividad física en estudiantes universitarios: diferencias en función del género, la edad y los estados de cambio. Revista Iberoamericana de Psicología del Ejercicioyel Deporte, 11(1), 123-132.

Puigarnau, S., Foguet, O. C., Balcells, M. C., Ambrós, Q. P., \& Anguera, M. T. (2016). El apoyo a la autonomía en practicantes de centros deportivos y de fitness para aumentar su motivación. RICYDE. Revista Internacional de Ciencias del Deporte, 12(43), 48-64. http://dx.doi.org/10.5232/ricyde2016.04303

Rodríguez M. F., Mata E., \& Ruiz L. M. (2006). El autoconcepto físico en la adolescencia temprana: influencia del género y la edad. Actas del I Congreso de Jóvenes Investigadores en Ciencias de la Actividad Física y del Deporte; 15 16 Dez 2006; Valladolid, España. Valladolid: CSD; 163-72.

Ryan, R. M. \& Deci, E. L. (2000). Self-determination theory and the facilitation on intrinsicmotivation, social development, and well-being. American Psychologist, 55(1), 68-78. http://dx.doi.org/10.1037/0003-066X.55.1.68

Sicilia, A., Águila, C., Muyor, J. Mª., Orta, A., \& Moreno, J. A. (2009). Perfiles motivacionales de los usuarios en centros deportivos municipales. Anales de Psicología, 25(1), 160-168

Sicilia,A., González-Cutre, D., Artés, E. M., Orta,A., Casimiro,A. J., \& Ferriz, R. (2014). Motivos de los ciudadanos para realizar ejercicio físico: un estudio desde la teoría de la autodeterminación. Revista Latinoamericana de Psicología, 46(2), 83-91.https://doi.org/10.1016/S0120-0534(14)70011-1

Sicilia, A., Ferriz-Morel, R., \& Coll, D. G. C. (2016). Relación entre la satisfacción delas necesidades psicológicas básicas durante la educación física recibida en la educación secundaria obligatoria y las conductas saludables al inicio del bachillerato. Revista Brasileira de Ciências do Esporte, 36(2), 59-74.

Standage, M. \& Gillison, F. (2007). Student's motivational responses toward school physical education and their relationship to general self-esteem and health-related quality of life. Psychology of Sport and Exercise, 8(5), 704-721. https://doi.org/10.1016/j.psychsport.2006.12.004

Texeira, P., Going, S., Houtkooper, L., Cussler, E., Metcale, L., Blew, R., Sardinha, L., \& Lohman, T. (2006). Exercise motivation, eating, and body image variables as predictors of weight control. Medicine \& Science in Sports \& Exercise, 38(1), 179-188.

Vallerand, R. J. (1997). Toward a hierarchical model of intrinsic and extrinsic motivation. En M. P. Zanna(Ed.), Advances in experimental social psychology (pp.271-360). Academic Press: New York.

Vallerand, R. J. \& Rousseau, F. L. (2001). Intrinsic and extrinsic motivation in sport and exercise: Areview using the hierarchical model of intrinsic and extrinsic motivation. En R. N. Singer, H. A. Hausenblas, y C. M. Janelle (Eds.), Handbook of sport psychology (pp. 389-416). New York: Wiley.

Wilson, P. M., Rodgers, W. M., Loitz, C. C., \& Scime, G. (2006). «It’s Who I Am... Really!. The Importance of Integrated Regulation in Exercise Contexts. Journal of Applied Biobehavioral Research, 11(2), 79-104. doi: 10.1111/j.1751-9861.2006.tb00021.x

Wilson, P. M., Rogers, W. T., Rodgers, W. M., \& Wild, T. C. (2006). The psychological need satisfaction in exercise scale. Journal of Sport and Exercise Psychology, 28(3), 231-251. https://doi.org/10.1123/jsep.28.3.231

Wold, B. (1995). Health behavior in school children: A WHO cross-national survey. Resource Package of Questions 1993-94. Bergen: University of Bergen.



